Conclusion After 12 weeks LOLA, patients reported highly significant improvement in energy levels and concentration. Although 12 weeks LOLA had no overall effect on psychometric performance, significant treatment-related improvement in digit-symbol PHES subtest in those receiving LOLA was seen. An increase was noted in biceps skinfold thickness, which may indicate improved nutrition. Subcortical brain areas showed volume reduction, an observation not previously noted in imaging studies of patients receiving this drug. Unlike previous studies, no functional changes were seen, but significant changes were found on MRS of ACC, a region known to be metabolically active in mHE. It may be that a larger dose of LOLA would have shown greater effects on psychometric performance.

\section{PWE-092 A STRATIFIED HOME BASED CARE PATHWAY FOR THE TREATMENT OF HCV INFECTION}

Brian Thomson*, Jasmina Khaldi, Samantha Bird, Tom Bills, Breanne Dilks, Stephen Ryder. Nottingham University Hospitals, Nottingham, UK

\subsection{6/gutjnl-2018-BSGAbstracts.234}

Introduction The advent of directly acting anti-viral agents (DAAs) for HCV infection has transformed the therapeutic landscape. DAAs have an excellent safety record, and can be stratified for use in community environments which better meet patient needs. We present a pilot study of a novel home based care pathway, delivered by a partnership between the local Operational Delivery Network (ODN), specialist hepatitis services and clinical Pharmacy.

Methods HCV infected patients assessed at the ODN multidisciplinary team meeting (MDT) as eligible for DAAs were screened for entry to the study by the following criteria: competence to adhere to therapy without direct supervision; no current or previous evidence of hepatic decompensation; contactable by telephone. Following recruitment, patients received a pack containing: blood forms; a schedule for community blood testing; information leaflets; details of hospital contacts. DAAs were delivered to the patient home each month by Pharmacy. A dedicated Pharmacy technician acted as initial contact for patient queries, with support from the lead nurse specialist. The homecare technician worked closely with the nursing team, the MDT co-ordinator and ODN Manager to co-ordinate the service. Lab Results were reviewed by the lead nurse. Patients were invited to report outcome measures and feedback using structured questionnaires.

Results Of 121 patients offered a choice of home or hospital based care by January 2018, 97 (80\%) elected to receive treatment at home. This group did not differ significantly in age, gender, HCV genotype or choice of DAA from those treated in the hospital clinic. 14 homecare patients met Fibroscan criteria for cirrhosis. Of the 97 patients so far started on treatment, 57 have completed, 31 achieved SVR and 4 have failed treatment. Three patients withdrew from the study for reasons unrelated to homecare and one transferred back to hospital care. 18 feedback questionnaires have been received from 56 so far sent (32\%). All respondents stated that the service had lived up to or exceeded expectations, and was particularly valued by patients living distant from the hospital. Conclusions Homecare provides a safe, transferable and scaleable treatment option which is preferred by patients. The strategy of pharmacy based implementation and economies of staff time intrinsic to the homecare model will relieve pressure on hepatitis services, and allow specialist teams to focus on patients with severe co-morbidities and promoting models for case finding and community care for harder to reach groups with HCV infection.

\section{PWE-093 TREATMENT OF GASTRIC FUNDAL VARICES WITH EUS GUIDED EMBOLISATION COMBINING COIL PLACEMENT WITH THROMBIN INJECTION}

${ }^{1} J o a n n e$ Orourke* ${ }^{1,2} \mathrm{Chander}$ Shekhar, ${ }^{1}$ Dhiraj Tripathi, ${ }^{1} \mathrm{Colm}$ Forde, ${ }^{1}$ Brinder Mahon. ${ }^{1}$ Queen Elizabeth Hospital, Birmingham, UK; ${ }^{2}$ Manor Hospital, Walsall, UK

\subsection{6/gutjnl-2018-BSGAbstracts.235}

Introduction Gastric varices are present in 5\%-33\% of patients with portal hypertension with incidence of bleeding of around $25 \%$ in 2 years. ${ }^{1}$ If gastric varices are identified as the source of bleeding, therapeutic options include endoscopic Methods, TIPSS, surgery and non-selective beta blockade. ${ }^{2}$ There are reports of EUS guided coiling combined with cyanoacrylate glue ${ }^{3}$ but limited literature on safety and efficacy of EUS guided coil embolisation with human thrombin injection. We report our experience.

Methods We analysed data of all EUS guided interventions for the management of bleeding gastric varices between 20152017 at a liver transplant centre. Olympus EUS linear scope was used to inject human thrombin (Tisseel; 500IU/ML) in gastric varices with or without coils (Nester Embolization Coils).

Results A total of 10 EUS guided interventions in 6 patients (4 $\mathrm{M}$ and $2 \mathrm{~F}$ ), aged 55 (41-59) yrs for secondary prophylaxis. $67 \%$ patients had cirrhosis with MELD score of 14(1021) and $75 \%$ were Child-Pugh class C. The remainder had non-cirrhotic portal hypertension. All patients had previous bleeding from gastric varices and $2 / 3$ rd were intolerant of beta-blockers. $67 \%$ had previous thrombin injection that had failed to obliterate the gastric varices. EUS guided coil embolisation was undertaken with thrombin injection in 6 , and thrombin alone in 4 ( 2 had previous coils embolisation). The largest feeding vessel was $12(7-16) \mathrm{mm}$ with a median 5 (210) coils placement followed by thrombin injection of 3500 (2500-5000) IU.

Most (8/10) stayed overnight after intervention and only 2 required longer stays, Median F/U was 9 (3-20) months with zero 30 day mortality. 1 patient had fever 2 days post procedure requiring IV antibiotics. No reported episodes of rebleeding except in 1 patient at 23 months. 4 had follow up EUS (5-7 months) and showed no flow at the level of the coils. 1 patient died within 3 months of procedure secondary to hepatic decompensation.

Conclusions In our experience EUS guided coil embolisation and injection of thrombin, is a technically safe and well-tolerated procedure even in patients with advanced liver disease especially who have failed eradication of gastric varices from single modality therapy. Due to the lower incidence of gastric variceal bleeding in comparison to oesophageal varices bleeding, we recommend multi-centre prospective data collection evaluating the modalities being used and reporting of outcomes to help inform national guidelines.

\section{REFERENCES}

1. Hepatology 1992;16:1343-1349.

2. Gut 2015;64:1680-704.

3. Gastrointest Endosc. 2016 Jun;83(6):1164-72. 